\title{
MATHEMATICAL MODELING OF COMPLEX MULTI-COMPONENT MOVEMENTS AND OPTICAL METHOD OF MEASUREMENT
}

\author{
V.N. Nesterov \\ JSC “Samara Electromechanical Plant”, Samara, Russia
}

\begin{abstract}
The provisions of the concept of a multi-component vector physical value are given, which base on an understanding as objects displacement processes structured complex processes and can be sources of various factors including independent ones. The multi-component model of moving objects motion containing informative components were presented in the general form. The problem of measuring these informative components was formulated and the fundamentals of the named optical measurement values were presented on the basis of the multi-dimensional method of testing facilities are presented.
\end{abstract}

Keywords: concept, models, multi-component displacements, optical measurement, image processing.

Citation: Nesterov VN. Mathematical modeling of complex multicomponent movements and optical method of measurement. CEUR Workshop Proceedings, 2016; 1638: 642-649. DOI: 10.18287/1613-0073-2016-1638-642-649

Questions about choice of calculation and measuring models of object have of key importance for the successful solution of the problem of measurement of informative components of complex movements of moving objects. The complexity of the objects and processes, leads to different in appearance and information content of displacements and deformations, and requires study and development of a common ideology of their modeling. The lack of united information and mathematical models of controlled processes complicates the qualitative and quantitative selection of measurement methods and tools, as well as the processing of the results.

If the monitored objects and associated processes have complex character and/or structure, that the movement, which is their consequence, himself will be characterized by a certain structure, elements of which are connected to each other in any way, they are in communication, have a mutual influence on each other and bear additional information about the studied phenomenon or object.

Understanding of the processes of displacement simple objects and even complex objects like complex structured processes, the sources of which may be different, including the independent factors, led to the realization of the need to formulate the concept of a multi-component vector physical quantity [1]. In this case, the concept is used to describe complex multi-movement of moving objects. 
The main provisions of the concept represented by the following three points [1-3]:

- Vector multi-component physical values were considered as functions of the set of their constituent informative components;

- The above mentioned informative components of communication functions in multi-component physical values models were determined by the apparatus of vector algebra;

- Information models of multi-component physical vector values allow multivariate representation of these informative components, depending on the object of study and the task.

Taking into account the above provisions mathematical model of vector multicomponent physical quantity it was written in a Cartesian coordinate system in general terms as:

$$
\left.\begin{array}{l}
\mathbf{X}_{x}(\mathbf{r}, \tau)=\mathbf{F}\left(\mathbf{x}_{1 x}(\mathbf{r}, \tau), \ldots, \mathbf{x}_{p x}(\mathbf{r}, \tau)\right) \\
\mathbf{X}_{y}(\mathbf{r}, \tau)=\mathbf{F}\left(\mathbf{x}_{1 y}(\mathbf{r}, \tau), \ldots, \mathbf{x}_{p y}(\mathbf{r}, \tau)\right) ; \\
\mathbf{X}_{z}(\mathbf{r}, \tau)=\mathbf{F}\left(\mathbf{x}_{1 z}(\mathbf{r}, \tau), \ldots, \mathbf{x}_{p z}(\mathbf{r}, \tau)\right),
\end{array}\right\}
$$

Here $\mathbf{X}_{x}(\mathbf{r}, \tau), \mathbf{X}_{y}(\mathbf{r}, \tau), \mathbf{X}_{z}(\mathbf{r}, \tau)$ are projections of multi-component displacement on the coordinate axes of the Cartesian coordinate system; $\mathbf{x}_{1 k}(\mathbf{r}, \tau), \ldots, \mathbf{x} p k(\mathbf{r}, \tau)$ are information components $k$-th component of the coordinate $(k \in\{x, y, z\})$ multicomponent displacement $\mathbf{X} ; \mathbf{r}, \tau$ are spatial and temporal coordinates; $\mathbf{F}$ is communication function which defined by the physics of the object or process.

According to the second paragraph referred to the provisions of the multi-component vector physical quantity model (1) is represented as a vector sum of the corresponding informative components:

$$
\left.\begin{array}{l}
\mathbf{X}_{x}(\mathbf{r}, \tau)=\sum_{j=1}^{p} \mathbf{x}_{j x}(\mathbf{r}, \tau) \\
\mathbf{X}_{y}(\mathbf{r}, \tau)=\sum_{j=1}^{p} \mathbf{x}_{j y}(\mathbf{r}, \tau) ; \\
\mathbf{X}_{z}(\mathbf{r}, \tau)=\sum_{j=1}^{p} \mathbf{x}_{j z}(\mathbf{r}, \tau),
\end{array}\right\}
$$

Here $\sum_{j=1}^{p} \mathbf{x}_{j x}(\mathbf{r}, \tau), \sum_{j=1}^{p} \mathbf{x}_{j y}(\mathbf{r}, \tau), \sum_{j=1}^{p} \mathbf{x}_{j z}(\mathbf{r}, \tau)$ are vector sum $p$ coordinate informative components constituting a multi-component movement $\mathbf{X}$.

Analysis of practical problems distinguished by complexity of controlled objects and tracks their movements in real three-dimensional space, has revealed the problem: 
- Formation of models and measurements of components of complex movements, which undergo simple objects;

- Formation of models and measurements of components of simple movements, which undergo complex objects;

- Formation of models and measurements of components of complex movements, which undergo complex objects.

These problems arise in the process of solving the problems associated with the design of optical systems determining motion parameters of moving objects, noncontact measurement systems parameters running part of vehicles in their diagnosis. Private and very complex problem of modeling the components moving parts of the manipulator appears in the calibration of universal industrial robots.

If we understand the relativity of movement of the various points of the controlled object, as well as the relativity of coordinate system, for example, when modeling the displacement of links universal industrial robot [4], we understand the need and importance of the third position the concept of vector multi-component physical value.

Illustration models of complex multi-component motion of moving objects are beyond the scope of this study.To understand the complexity of the latter can refer to $[4,5]$. We illustrate the problem of the relatively simple task of multi-component motion of a simple object.

Figure 1 was shown the decomposition of multi-component moving deformable object, made in the form of a circle. The object moved from the position I to III of the situation, while turning around an axis perpendicular to the plane of drawing and tied to the position $\mathrm{O}_{i}$ was taken as a pole. At the same time the object changed its geometrical parameters, such as $\Delta r=r_{0}-r_{1}$.

By decomposition of movement points $\mathrm{A}$ and $\mathrm{B}$ of the object projected onto the coordinate axis OX, following the next informative elements: $x_{1}$ is the component that characterized the movement of point $\mathrm{O}_{i}$ of the object, taken as a pole in the direction of the coordinate axis OX; $\mathbf{x}_{2}, \mathbf{x}_{2}^{\prime}$ are components of the movement points $\mathrm{A}$ and $\mathrm{B}$, describing the object rotates around a coordinate axis passing through the pole $\mathrm{O}_{i} ; \mathbf{x}_{3}, \mathbf{x}_{3}^{\prime}$ are components characterizing the deformation of object in the radius direction.Then the projections of points $\mathrm{A}$ and $\mathrm{B}$ on the axis OX can make a model containing three components, each isformative:

$$
\begin{aligned}
& \mathbf{X}_{1}=\mathbf{x}_{1}+\mathbf{x}_{2}+\mathbf{x}_{3}, \\
& \mathbf{X}_{2}=\mathbf{x}_{1}+\mathbf{x}_{2}^{\prime}+\mathbf{x}_{3}^{\prime} .
\end{aligned}
$$

Informative components recording in the right parts of the model (3) and (4), characterizing by different sources, the impact or effect of which leads to a resulting change in the multi-component physical values on the right sides of (3) and (4), and, thus, represent a significant interest. At the same time, in accordance with the concept of $[1,2]$ components included in the multi-component physical value have a physical dimension, and that the value as a whole, substantially or fully overlapping spectral 
range coinciding. Recent circumstances significantly complicate the process of measuring these components, making it impossible using the known measuring methods for their determination.

So we have models, and we have variety of informative components combined by these models in a multi-component value. There remains to choose the method of measurement and the corresponding measuring instruments. The most promising in that case is an optical method [6]. But there occurs a twofold problem: firstly, as already mentioned, the non-selectivity of measuring instruments in general, and optical in particular in respect to the informative components to be measured; and secondly, the incorrectness of recovering the object coordinates problem in real space on the 2D image basis. The latter is illustrated in Figure 2.

The relationship between the coordinates of the point $m\left(x_{0}, y_{0}\right)$ which is the image of a real object point is given by the system of two equations with three unknown variables:

$$
\frac{x_{0}}{\lambda}=\frac{X_{1}}{\lambda-Z_{1}} ; \quad \frac{y_{0}}{\lambda}=\frac{Y_{1}}{\lambda-Z_{1}},
$$

where $\lambda$ is the focal length of the lens.

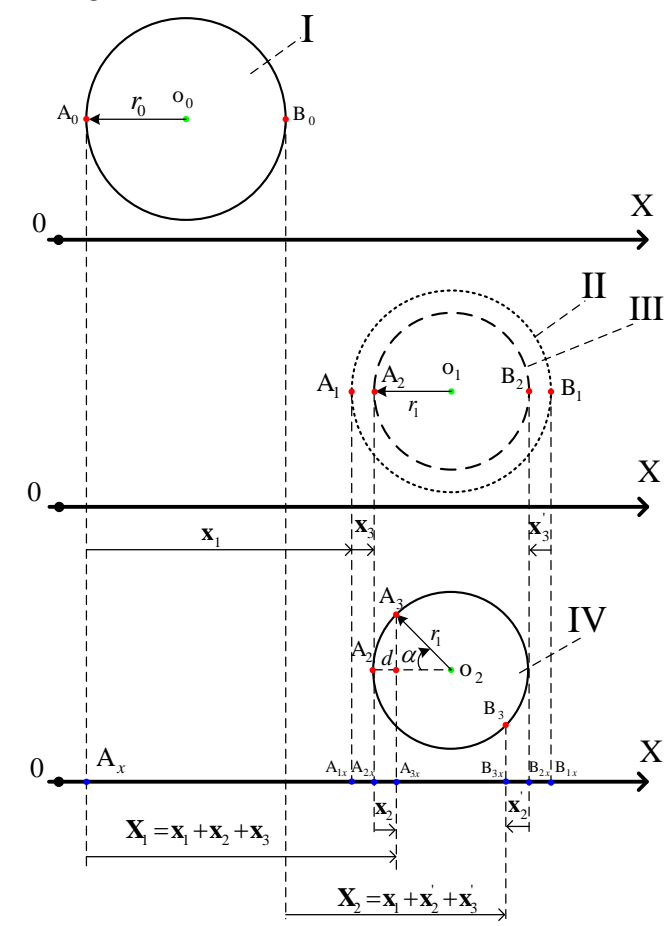

Fig. 1. Decomposition of multi-component moving deformable object 


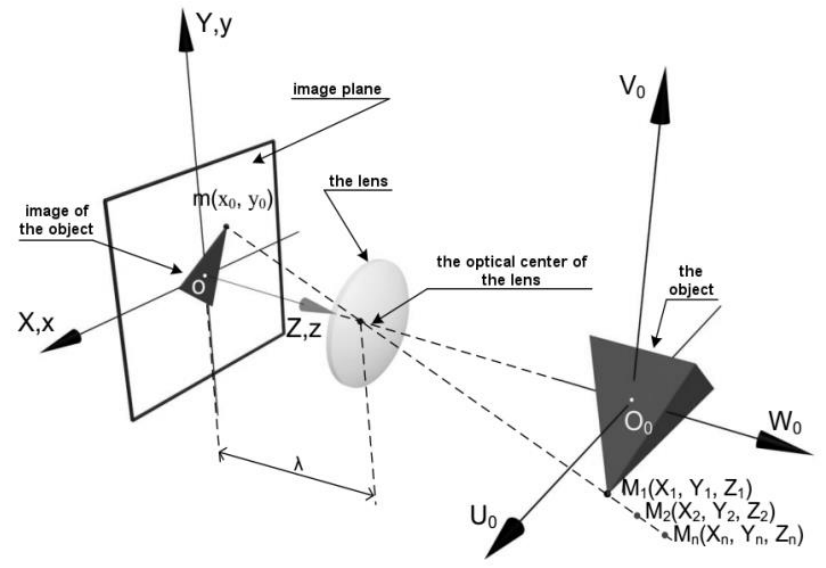

Fig. 2. Example of image formation

All points lying on a straight line passing through the points with coordinates $\left(x_{0}, y_{0}, 0\right)$ and $(0,0, \lambda)$ are projected in the image point $m\left(x_{0}, y_{0}\right)$.

As a rule these problems are solved by providing information redundancy in the measuring system. For example, the binocular vision systems can be used for computer vision [4]. The original solution proposed in the present article is based on the use of the visually controlled space distributed test object with geometric parameters known with high precision, which are used as measures [7].

The essence of the method of multi-dimensional test object boils down to that in order to measure the informative components of movement of the controlled object by an optical method with the object binds the distributed object in space having known with high precision the geometric parameters, which are used in the process of implementation of the method as the measures.In the particular case of a controlled object directly it can be used as a test object.

A special feature of the method is that the parameters of a multi-dimensional test object reflect the multidimensionality of controlled movements and functionallylink to them in the process of formation of models for further implementation of the method. Accordingly, the mathematical objects $\mathbf{L}_{1 k}, \ldots, \mathbf{L}_{q k}$ are introduced to model (1) duethe using of a new physical object, called test [7]:

$$
\left.\begin{array}{r}
\mathbf{X}_{x}(\mathbf{r}, \tau)=\mathbf{F}\left(\mathbf{x}_{1 x}(\mathbf{r}, \tau), \ldots, \mathbf{x}_{p x}(\mathbf{r}, \tau), \mathbf{L}_{1 x}, \ldots, \mathbf{L}_{q x}\right) ; \\
\mathbf{X}_{y}(\mathbf{r}, \tau)=\mathbf{F}\left(\mathbf{x}_{1 y}(\mathbf{r}, \tau), \ldots, \mathbf{x}_{p y}(\mathbf{r}, \tau), \mathbf{L}_{1 y}, \ldots, \mathbf{L}_{q y}\right) ; \\
\mathbf{X}_{z}(\mathbf{r}, \tau)=\mathbf{F}\left(\mathbf{x}_{1 z}(\mathbf{r}, \tau), \ldots, \mathbf{x}_{p z}(\mathbf{r}, \tau), \mathbf{L}_{1 z}, \ldots, \mathbf{L}_{q z}\right),
\end{array}\right\}
$$

where $\mathbf{L}_{1 k}, \ldots, \mathbf{L}_{q k}$ are components $k$ of coordinate component $\mathbf{L}_{k}$ of a multidimensional test object (multivariate test) $\mathbf{L} ; q$ is the number of component $k$ of 
coordinate component $\mathbf{L}_{k}$ of a multi-dimensional test object (multivariate test) $\mathbf{L} ; \mathbf{F}$ are component communication functions.

Signs of forming the method of multi-dimensional test objects which distinguish this method of measurement of other known and enable to fulfill the conditions of its physical realization are formulated in a number of policy papers [6,7] and are as follows.

1. The presence (the ability to form) the system of $n$ equations, dissymmetric with respect to the informative components $\mathbf{x}_{1 k}(\mathbf{r}, \tau), \ldots, \mathbf{x}_{p k}(\mathbf{r}, \tau)(k \in\{x, y, z\}$ is the set of coordinate components movements of relevant test object image points):

$$
\begin{aligned}
& \mathbf{Y}_{1}(\mathbf{r}, \tau)=\Psi_{1}\left\{\mathbf{F}_{1}\left\{\mathbf{x}_{1 k}(\mathbf{r}, \tau), \ldots, \mathbf{x}_{p k}(\mathbf{r}, \tau), \mathbf{L}_{1 k}, \ldots, \mathbf{L}_{q k}\right\}\right\} \\
& \mathbf{Y}_{n}(\mathbf{r}, \tau)=\Psi_{n}\left\{\mathbf{F}_{p}\left\{\mathbf{x}_{1 k}(\mathbf{r}, \tau), \ldots, \mathbf{x}_{p k}(\mathbf{r}, \tau), \mathbf{L}_{1 k}, \ldots, \mathbf{L}_{q k}\right\}\right\}, \\
& (n \geq p \geq 2) \text {, } \\
& \mathbf{F}_{1}\left\{\mathbf{x}_{1 k}(\mathbf{r}, \tau), \ldots, \mathbf{x}_{p k}(\mathbf{r}, \tau), \mathbf{L}_{1 k}, \ldots, \mathbf{L}_{q k}\right\} \neq \ldots \neq \mathbf{F}_{p}\left\{\mathbf{x}_{1 k}(\mathbf{r}, \tau), \ldots, \mathbf{x}_{p k}(\mathbf{r}, \tau), \mathbf{L}_{1 k}, \ldots, \mathbf{L}_{q k}\right\},
\end{aligned}
$$

where $\mathbf{Y}_{1}(\mathbf{r}, \tau), \ldots, \mathbf{Y}_{n}(\mathbf{r}, \tau)$ - displacement functions of the corresponding image points of the controlled object with respect to selected reference point (s) of the image; $\mathbf{F}_{1}\left\{\mathbf{x}_{1 k}(\mathbf{r}, \tau), \ldots, \mathbf{x}_{p k}(\mathbf{r}, \tau), \mathbf{L}_{1 k}, \ldots, \mathbf{L}_{q k}\right\}, \ldots, \mathbf{F}_{p}\left\{\mathbf{x}_{1 k}(\mathbf{r}, \tau), \ldots, \mathbf{x}_{p k}(\mathbf{r}, \tau), \mathbf{L}_{1 k}, \ldots, \mathbf{L}_{q k}\right\}$ are vector set functions of their constituent informative components $\mathbf{x}_{1 k}(\mathbf{r}, \tau), \ldots, \mathbf{x}_{p k}(\mathbf{r}, \tau)$ and components $\mathbf{L}_{1 k}, \ldots, \mathbf{L}_{q k} k$ of coordinate component $\mathbf{L}_{k}$ of a multi-dimensional test object (multivariate test) $\mathbf{L}$.

2. The feasibility of special measuring and computing algorithms is:

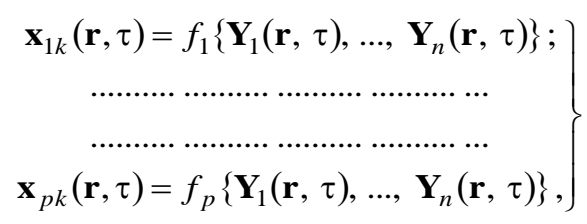

the existence condition for of which, with continuity and differentiability of $\mathbf{Y}_{1}(\mathbf{r}, \tau), \ldots, \mathbf{Y}_{n}(\mathbf{r}, \tau)$ in the whole range of measurement, is:

$\operatorname{det}\left[\frac{\partial \mathbf{Y}_{i}(\mathbf{r}, \tau)}{\partial \mathbf{x}_{j k}(\mathbf{r}, \tau)}\right] \neq 0 \quad i=\overline{1, n}, \quad j=\overline{1, p}$.

Condition (10) is ensured by the implementation of the "dissymmetry" values $\mathbf{Y}_{1}(\mathbf{r}, \tau), \ldots, \mathbf{Y}_{n}(\mathbf{r}, \tau)$ respecting to their components $\mathbf{x}_{1 k}(\mathbf{r}, \tau), \ldots, \mathbf{x}_{p k}(\mathbf{r}, \tau)$ and $\mathbf{L}_{1 k}, \ldots, \mathbf{L}_{q k}$, which is expressed by the inequality (8). 
When using a single-channel optical system the functions $\psi_{1}, \ldots, \psi_{n}$ are the same. Then for the model (7) they can be described as an optical transducer transfer coefficient $\sigma$ :

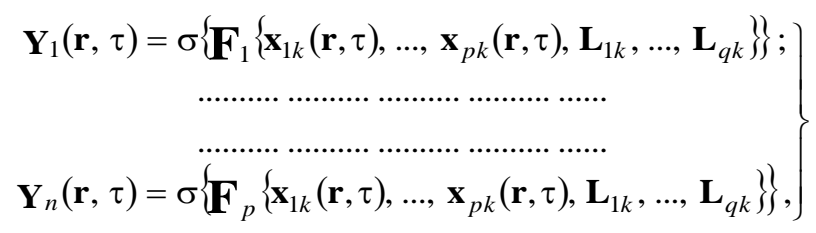

$(n \geq p \geq 2)$.

The main feature of the multi-dimensional test objects method, the name of which is essentially based on the understanding of its fundamental importance is not only providing more information on the input, but also the existence of original instrument for building in multivariate test objects parameters in the multi-displacement models generated within the frames of the method realization. According to the importance and the logic of the present scientific direction, development of the apparatus for mathematical models formal synthesis of multicomponent displacements and deformations of moving objects with build in parameters of test objects can be placed on the second place immediately after the vector multi-component physical values conception.

The most complete and fundamental summary of the optical measurements theory based on the multi-dimensional test objects is presented in the recent monograph [6]. Here are compound together published at different times in different scientific and technical journals and conference proceedings foundations of the concept of vector multi-component physical values, the apparatus for the formal synthesis of multicomponent models displacements of moving objects as a logical extension of the conception, theoretical concepts and practical results of the optical measurements of the informative components of moving objects multicomponent displacements and deformations based on the multivariate test objects. Also is shown the method performance. The good results obtained in spite of the low measurement accuracy of the used in consolidated measurement system means are cheering. This fact allows hoping for increasing the systems measurement metrological characteristics based on precision components.

The sequence of actions, implementation of which allows realizing optical measurements of the moving objects multi-component displacements on the basis of the observed method is shown in the form of an algorithm, the description of which is also given in [6]. In general it was possible to avoid such an algorithm. But considering many difficult for understanding and implementation requirements which must be fulfilled both on problem formulation stage and in the process of method physical feasibility, it was decided to keep this algorithm. The special attention should be paid to the particular importance and ambiguity of the integration procedures of the formed mathematical models according to the requirements of the equations system method. The formal instrument for that type of models synthesis allows implementing the 
large options variety meeting the criterion of equations systems solvability (11) relatively to the desired informative components. In [6] this fact is shown in many examples. However, as a fly in the ointment, there arises the problem of choosing from a variety of quasiequal options, the solution of which has to be found.

\section{References}

1. Nesterov VN. Theoretical basis of measurement components of vector multi-component physical values. Proceedings of the III International Conference "System Identification and Control Problems". Institute of Control Problems behalf of V.A. Trapeznikov, Russian Academy of Sciences, 2004: 1691-1700.

2. Nesterov VN. Theoretical Principles for Measuring the Components of a Vector Physical Quantity. Measurement Techniques, 2004; 47(7): 657-664.

3. Nesterov VN, Meshchanov AV. Models for vector multicomponent physical quantities and a multivariate test method for optical measurement systems. Measurement Techniques, 2006; 49(12): 1182-1188.

4. Fu K, Gonzalez P, Li K. Robotic. M.: Mir, 1989; 624 p.

5. Nesterov VN, Zherebyatiev KV. Mathematical modeling of the six-membered universal manipulator of an industrial robot. Direct kinematic problem for a robot PR125. Vestnik of Samara State Technical University. Series Engineering, 2005; 32: 19-28.

6. Nesterov VN, Mukhin VM, Meshchanov AV. The method of multi-dimensional test objects in optical measuring systems. Ed. V.N. Nesterov. Samara: Publishing House of the Samara Scientific Center of RAS, 2013; 224 p.

7. Nesterov VN, Meshchanov AV. Theoretical principles of optical measurements on the components of multicomponent displacements for mobile objects on the basis of multivariate tests. Measurement Techniques, 2007; 50(11): 1127-1136. 